\title{
Ocorrência de hemoglobina $S$ no estado de Mato Grosso do Sul, Brasil
}

\author{
Occurrence of hemoglobin S in Mato Grosso do Sul state, Brazil
}

Denise Rodrigues Holsbach'; Maria Lúcia Ivo²; Michael Robin Honer; Leonardo Rigo4; Carlos Augusto de Oliveira Botelho ${ }^{5}$

unitermos
Triagem neonatal
Hemoglobinopatias
Traço falciforme

\section{resumo}

Introdução: As hemoglobinopatias são as alterações genéticas mais comuns no homem, sendo a hemoglobina $(\mathrm{Hb}) \mathrm{S}$ a mais freqüente entre todas. Sua ocorrência no estado de Mato Grosso do Sul ainda não foi sistematicamente avaliada. Objetivos: Caracterizar a ocorrência de Hb S por genótipos, sexo, idade no momento do diagnóstico, índice de cobertura e prevalência em Mato Grosso do Sul. Material e método: Estudo retrospectivo, transversal e descritivo, desenvolvido com os resultados de triagem neonatal para hemoglobinopatias, utilizando a técnica de cromatografia líquida de alta pressão, no Instituto de Pesquisas, Ensino e Diagnósticos da Associação de Pais e Amigos dos Excepcionais (IPED/APAE) de Mato Grosso do Sul em 2000-2005. Resultados: De 190.809 indivíduos triados, 2.624 (1,38\%) encontraramse alterados, correspondendo a 2.385 neonatos e 239 crianças maiores de 28 dias. Não houve diferença entre os sexos, sendo 1.335 do sexo feminino e 1.289 do masculino. Os genótipos alterados encontrados foram traço falciforme (FAS [99,16\%]) e doenças falciformes (FS [0,61\%] e FSC [0,23\%]). Conclusão: Esse primeiro estudo de triagem realizado no estado de Mato Grosso do Sul mostra que o programa desenvolvido pelo IPED/APAE está se solidificando no estado e avançando em relação ao índice de cobertura da população e ao diagnóstico precoce. Esses indicadores podem embasar ações preventivas (aconselhamento genético e estudos familiares) e assistenciais (tratamento ambulatorial contínuo), que visam à redução da morbimortalidade de indivíduos acometidos por essas afecções no estado.

\section{abstract}

Background: Hemoglobinopathies are the most common genetic disorders in humans and $\mathrm{Hb} \mathrm{S}$ is the most frequent among them. Its occurrence in the state of Mato Grosso do Sul has not been systematically analyzed yet. Objectives: To describe the occurrence of hemoglobin S according to genotypes, gender, age at the moment of diagnosis, cover index and prevalence in the state of Mato Grosso do Sul. Material and method: Retrospective, transversal and descriptive study of the results of neonatal screening for hemoglobinopathies performed with high pressure liquid chromatography technique at Instituto de Pesquisa, Ensino e Diagnósticos da Associação dos Pais e Amigos dos Excepcionais (IPED/APAE) in the state of Mato Grosso do Sul (20002005). Results: Among 190,809 screened individuals, 2,624 (1.38\%) showed alterations, 2,385 were neonates and 239 were children aged 28 days or more. There was no difference in gender (1,335 females and 1,289 males). The altered genotypes were FAS (99.16\%), FS (0.61\%) and FSC (0.23\%). Conclusion: This first study of neonatal screening in the state of Mato Grosso do Sul revealed that the state program developed by IPED/APAE has been consolidating and advancing as to cover index and early diagnosis. These indicators may be the basis for preventive (genetic counseling and family studies) and assistance measures (continuous ambulatory treatment), which aim at the reduction of morbimortality in individuals with these hemoglobinopathies in the state. key words

Neonatal screening

Hemoglobinopathies

Sickle cell trait

1. Mestra em Saúde Coletiva pela Universidade Federal de Mato Grosso do Sul (UFMS); docente do curso de Fisioterapia da Faculdade Estácio de Sá de Campo Grande (MS).

2. Doutora em Enfermagem pela Escola de Enfermagem de Ribeirão Preto da Universidade de São Paulo (EERP/USP); docente do curso de mestrado em Saúde Coletiva na UFMS.

3. Doutor em Epidemiologia e Ecologia pela Universidade de Utrecht, Holanda; docente no curso de mestrado em Biotecnologia da Universidade Católica Dom Bosco (UCDB).

4. Doutor em Ciências da Saúde pela Universidade de Braślia (UnB); coordenador de informações de saúde da Secretaria de Saúde (SESAU).

5. Biólogo; superintendente do Instituto de Pesquisas, Ensino e Diagnósticos da Associação de Pais e Amigos dos Excepcionais (IPED/APAE).

Artigo original baseado em dissertação de mestrado intitulada "Epidemiologia da anemia falciforme no estado de Mato Grosso do Sul, 2000-2005", defendida em 3 de julho de 2007 na UFMS. 


\section{Introdução}

As alterações dos genes das globinas causam os defeitos hereditários da síntese da hemoglobina ( $\mathrm{Hb})$. A mutação que resulta em $\mathrm{Hb} \mathrm{S}$ se dá pela troca de uma única base no códon 6 do gene da globina â. Uma adenina é substituída por uma timina, resultando na troca do resíduo glutamil na posição beta 6 por um resíduo valil(9). Essa modificação estrutural dos aminoácidos manifesta seu efeito por meio de precipitação e polimerização da $\mathrm{Hb} \mathrm{S}$, acarretando mudança de forma, o que conduz à deformação da hemácia, que adquire a forma de foice ${ }^{(13)}$, reduzindo drasticamente o tempo de vida da célula para sete a 25 dias ${ }^{(15)}$.

O termo "doença falciforme" é usado para determinar um grupo de afecções genéticas caracterizadas pelo predomínio da $\mathrm{Hb} \mathrm{S}$. Essas alterações incluem a anemia falciforme (Hb SS) e as duplas heterozigoses, como HbSC ${ }^{(11)}$. A heterozigose para $\mathrm{Hb} \mathrm{S}$ define uma situação clinicamente benigna em que o indivíduo apresenta as hemoglobinas $\mathrm{A}$ e $S(\mathrm{HbAS})^{(2)}$.

A inclusão obrigatória da pesquisa de hemoglobinopatias no exame de triagem neonatal, regulamentada pela Portaria no 822, de 6 de junho de 2001, do Ministério da Saúde ${ }^{(8)}$, é relevante para a redução da taxa de mortalidade entre crianças. Isso permite precoce identificação das alterações, profilaxia adequada e seguimento ambulatorial regular ${ }^{(20)}$.

Em janeiro do ano 2000, a Lei Estadual no 2.079 tornou compulsória a realização de exames para detecção de hemoglobinopatias em crianças recém-nascidas em todo o estado de Mato Grosso do Sul(12).

A triagem neonatal realizada pelo Instituto de Pesquisas, Ensino e Diagnósticos da Associação de Pais e Amigos dos Excepcionais (IPED/APAE) no estado de Mato Grosso do Sul utiliza o método diagnóstico de cromotografia líquida de alta eficiência (HPLC), que, segundo Araújo et al.(3), é considerado técnica altamente sensível para o estudo das hemoglobinas anormais por evidenciar pequenas quantidades de $\mathrm{Hb} \mathrm{A}$, bem como $\mathrm{Hb} \mathrm{S}, \mathrm{Hb} \mathrm{C}$ e outras hemoglobinas anormais, na presença de grandes quantidades de $\mathrm{Hb} F$. Os sistemas automatizados proporcionam um processamento rápido das amostras com alto índice de reprodutibilidade e exatidão, constituindo-se um excelente método para triagem neonatal.

Naoum e Naoum ${ }^{(15)}$ referem ainda que, na automação da HPLC, há grande eficiência e precisão, permitindo a quantificação de $\mathrm{Hb}$ A, A2 e fetal, bem como de hemoglo- binas variantes. Assim, as hemoglobinas A, A2, fetal, S, C, O Arábia, D Los Angeles e $G$ Filadélfia podem ser separadas umas das outras.

Considerando os aspectos mencionados, pretende-se neste estudo caracterizar a ocorrência de HbS por genótipos, sexo, idade em dias no momento do diagnóstico, índice de cobertura e prevalência no estado de Mato Grosso do Sul no período de 2000 a 2005.

\section{Material e método}

Estudo retrospectivo, transversal e descritivo desenvolvido a partir dos resultados obtidos na triagem neonatal para hemoglobinopatias no IPED-APAE, em Mato Grosso do Sul, no período 2000-2005.

A amostra geral consistiu em 190.809 indivíduos submetidos a exame para identificação de hemoglobinopatias pelo Programa Nacional de Triagem Neonatal (PNTN) desenvolvido no estado de Mato Grosso do Sul. Foram incluídas nos resultados todas as pessoas que apresentaram o diagnóstico de doença falciforme ou traço falciforme comprovado por HPLC. Tal metodologia é automatizada e utiliza kits para confirmação e reconfirmação dos casos alterados, ambos do BIO-RAD Laboratories ${ }^{\circledR}$.

Foram excluídos aqueles que possuíam outras hemoglobinopatias não-falciformes ou tinham informações não-disponíveis em sua ficha, o que se tornou um fator de limitação da pesquisa, uma vez que se utilizaram dados secundários.

As variáveis selecionadas foram sexo, idade no momento do diagnóstico e índice de cobertura. A variável idade (em dias) foi categorizada em três faixas: 0 a 28, 29 a 180 e > 180 dias.

Os dados foram organizados em planilha Exce ${ }^{\circledR} \mathrm{e}$ as medidas descritivas calculadas com o programa SAS para Windows versão 8.0. As prevalências foram estimadas por ponto e por intervalo de confiança (IC) de $95 \%$, utilizando a distribuição de $\mathrm{F}$ e considerando a população finita ${ }^{(22)}$. Os dados acerca do tamanho da população foram os relatados no Sistema de Informações de Nascidos Vivos (SINASC).

O projeto de pesquisa, protocolo oㅡ 676, previamente aprovado pelo Comitê de Ética em Pesquisa da Universidade Federal de Mato Grosso do Sul (UFMS) em 14 de fevereiro de 2006, foi autorizado pela direção do IPED/APAE e desenvolvido integralmente segundo os princípios éticos estabelecidos na Resolução no 196, de 10 de outubro de 1996, do Conselho Nacional de Saúde (CNS). 


\section{Resultados}

$\mathrm{O}$ índice de cobertura de exames para hemoglobinopatias do PNTN em Mato Grosso do Sul variou durante o período de 2000 a 2005, destacando-se o ano 2002 com o maior índice (92,06\%) (Tabela 1). $O$ ano da implantação do programa, 2000, foi o que apresentou menor índice $(28,38 \%)$.

Foram analisados os resultados obtidos pelo IPED/APAE referentes a 190.809 indivíduos submetidos ao PNTN no estado de Mato Grosso do Sul no período de 2000 a 2005. Desse total, 2.624 (1,38\%) apresentaram $\mathrm{HbS}$ em diferentes associações genotípicas, sendo 2.385 neonatos ( 0 -28 dias) e 239 crianças (acima de 28 dias), sendo 1.335 do sexo feminino e 1.289 do masculino. Ao analisar a prevalência de traço falciforme (FAS) por ano, a Tabela $\mathbf{2}$ mostra que houve maior prevalência no ano 2000 em relação aos demais anos, provavelmente devido à baixa cobertura no início do programa, quando se destacaram os casos encontrados.

A freqüência dos diferentes genótipos observados foi: a) associação entre $\mathrm{Hb}$ fetal, $\mathrm{HbA}$ e $\mathrm{HbS}$ (FAS) - 99,16\%

\section{Total de triagens, exames de hemoglobinas e índice de cobertura em pessoas triadas no}

Tabela 1 IPED/APAE, Mato Grosso do Sul, 2000 a 2005

\begin{tabular}{|c|c|c|c|c|}
\hline \multicolumn{5}{|c|}{ Coleta de dados - IPED/APAE } \\
\hline Ano & Total de triagens & $\begin{array}{l}\text { Exames de } \\
\text { hemoglobinas }\end{array}$ & Total de nascidos vivos* & Índice de cobertura (\%) \\
\hline 2000 & 31.544 & 11.490 & 40.490 & 28,38 \\
\hline 2001 & 32.681 & 32.681 & 40.070 & 81,56 \\
\hline 2002 & 36.764 & 36.764 & 39.933 & 92,06 \\
\hline 2003 & 34.305 & 34.305 & 39.248 & 87,41 \\
\hline 2004 & 37.961 & 37.961 & 41.567 & 91,32 \\
\hline 2005 & 37.608 & 37.608 & 41.424 & 90,78 \\
\hline Total & 210.863 & 190.809 & 242.732 & - \\
\hline
\end{tabular}

*Informações Sistema de Informações de Nascidos Vivos do Departamento de Informação e Informática do Sistema Único de Saúde de Mato Grosso do Sul (SINASC/DATASUS/MS). IPED/APAE: Instituto de Pesquisas, Ensino e Diagnósticos da Associação de Pais e Amigos dos Excepcionais.

\section{Prevalências de FAS e FS e FSC estimadas por ponto e por intervalo de confiança de $95 \%$ em}

Tabela 2 pessoas triadas no IPED/APAE, Mato Grosso do Sul, 2000 a 2005

\begin{tabular}{|c|c|c|c|c|}
\hline \multicolumn{5}{|c|}{ FAS } \\
\hline Ano & Prevalência (\%)* & IC $95 \%$ ** & Prevalência (razão) & IC $95 \%$ \\
\hline 2000 & 2,463 & $2,2291-2,7170$ & $1 / 40,6$ & $1 / 44,9-1 / 36,8$ \\
\hline 2001 & 1,5881 & $1,5302-1,6488$ & $1 / 63$ & $1 / 65,4-1 / 60,6$ \\
\hline 2002 & 1,4688 & $1,4339-1,505$ & $1 / 68,1$ & $1 / 69,7-1 / 66,4$ \\
\hline 2003 & 1,7957 & $1,7458-1,8476$ & $1 / 55,7$ & $1 / 57,3-1 / 54,1$ \\
\hline 2004 & 1,5542 & $1,5174-1,5924$ & $1 / 64,3$ & $1 / 65,9-1 / 62,8$ \\
\hline 2005 & 1,5848 & $1,5463-1,6247$ & $1 / 63,1$ & $1 / 64,7-1 / 61,6$ \\
\hline Todo & 1,6477 & $1,6214-1,6746$ & $1 / 60,7$ & $1 / 61,7-1 / 59,7$ \\
\hline \multicolumn{5}{|c|}{ FS } \\
\hline & Prevalência (\%) & IC 95\% & Prevalência (razão) & IC 95\% \\
\hline Todo & 0,0105 & 0,0085-0,0131 & $1 / 9.540,5$ & $1 / 11.828,4-1 / 7.621,5$ \\
\hline \multicolumn{5}{|c|}{ FSC } \\
\hline & Prevalência (\%) & IC 95\% & Prevalência (razão) & IC $95 \%$ \\
\hline Todo & 0,0042 & $0,0029-0,0061$ & $1 / 23.851,1$ & $1 / 33.899,6-1 / 16.462,7$ \\
\hline
\end{tabular}


(2.602 casos); b) associação entre $\mathrm{Hb}$ fetal e $\mathrm{HbS}$ (FS) - 0,61\% (16 casos); e c) associação entre $\mathrm{Hb}$ fetal, $\mathrm{HbS}$ e $\mathrm{HbC}$ (FSC) - 0,23\% (seis casos). Considerando apenas os recém-nascidos ( 0 -28 dias), foram encontrados 19 neonatos com genótipos sugestivos de doenças falciformes (13 com FS e seis com FSC) e 2.366 com genótipos sugestivos de traço falciforme (FAS) (Tabelas 2 e $\mathbf{3}$ ).
Por outro lado, um estudo epidemiológico sobre doenças falciformes realizado em um hospital da Índia central constatou que a ocorrência no sexo feminino é menor por dispensarem-se, nesse país, melhores cuidados a indivíduos do sexo masculino, negligenciando os do feminino ${ }^{(14)}$. No Brasil, em Fortaleza, um estudo de triagem neonatal para hemoglobinopatias demonstrou maior prevalência de $\mathrm{HbS}$ no sexo masculino(16).

Tabela 3

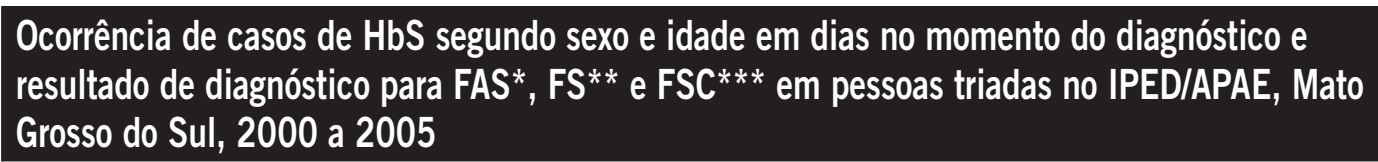

\begin{tabular}{|c|c|c|c|c|}
\hline \multicolumn{5}{|c|}{ Feminino } \\
\hline \multicolumn{5}{|c|}{ Diagnóstico } \\
\hline Idade (dias) & FAS & FS & FSC & Total \\
\hline 28 |---| 00 (0) & 1.210 & 6 & 4 & 1.220 \\
\hline 180 & 102 & 3 & 0 & 105 \\
\hline (2) $>180$ & 10 & 0 & 0 & 10 \\
\hline Total & 1.322 & 9 & 4 & 1.335 \\
\hline \multicolumn{5}{|c|}{ Masculino } \\
\hline \multicolumn{5}{|c|}{ Diagnóstico } \\
\hline Idade (dias) & FAS & FS & FSC & Total \\
\hline 28 |---| 00 (0) & 1.156 & 7 & 2 & 1.165 \\
\hline 180 & 117 & 0 & 0 & 117 \\
\hline$(2)>180$ & 7 & 0 & 0 & 7 \\
\hline Total & 1.280 & 7 & 2 & 1.289 \\
\hline
\end{tabular}

*Associação entre $\mathrm{Hb}$ fetal, $\mathrm{HbA}$ (normal) e HbS; **associação entre $\mathrm{Hb}$ fetal e $\mathrm{HbS}$; **aassociação entre Hb fetal, $\mathrm{HbS}$ e HbC.

FAS: traço falciforme; FS e FSC: doenças falciformes; Hb: hemoglobina.

\section{Discussão}

Os resultados mostraram que 2.624 pacientes analisados apresentaram hemoglobinopatias falciformes, caracterizando 1,38\% de alterações para o gene S. Esse achado corrobora os descritos na literatura, que mostram que a $\mathrm{Hb}$ S é, no Brasil, a mais freqüente das hemoglobinas variantes $(3,4)$.

Com relação ao sexo, os achados do presente estudo se assemelham à pesquisa epidemiológica conduzida por Bandeira et al.(5), que avaliaram, em Pernambuco, a prevalência de $\mathrm{HbS}$ e sua ocorrência segundo sexo, peso e Apgar, sem que encontrassem diferenças estatisticamente significativas entre sujeitos do sexo masculino e do feminino. Um estudo de triagem de hemoglobinopatias na cidade de Salvador tampouco encontrou diferença entre sujeitos do sexo masculino e do feminino(1).
Com relação à idade no momento do diagnóstico das hemoglobinopatias falciformes, houve destaque, nesta investigação, para os neonatos. O diagnóstico precoce éfundamental por causa da freqüência das hemoglobinopatias no Brasil e da gravidade de certas formas dessa patologia. Assim, a triagem neonatal e o diagnóstico precoce trazem benefícios essenciais à criação de programas preventivos e assistenciais( ${ }^{(18)}$. VianaBaracioli et al. ${ }^{(20)}$ compartilham dessas idéias e afirmam que, quanto mais precoce o diagnóstico, principalmente de homozigose, o acompanhamento pode ser iniciado, aumentando a sobrevida do paciente. Uma análise dos óbitos registrados pelo Sistema de Informações de Mortalidade, no período de 1979 a 1995, mostra que $25 \%$ dos portadores de anemia falciforme não alcançaram os 4 anos de idade, e quase $80 \%$ deles não completaram 30 anos ${ }^{(17)}$.

Nos últimos anos, as expectativas relativas à morbidade e à mortalidade pela doença falciforme modificaram-se significativamente, em parte devido à maior precisão e à 
precocidade no diagnóstico, e em parte ao crescente volume de novos conhecimentos sobre a doença. Além disso, houve gradual sensibilidade dos órgãos de saúde pública em nosso país, motivada especialmente por movimentos sociais relacionados com a população negra e as associações de portadores do gene $\mathrm{HbS}^{(15)}$.

Quanto aos índices de cobertura do PNTN em Mato Grosso do Sul, observa-se que a sua implantação ocorreu em 2000, com discreto aumento em 2002 e posterior decréscimo, seguida por discreta variação no período pesquisado, evidenciando que o programa desenvolvido pelo IPED/APAE ainda está se solidificando no estado. Este está avançando em relação ao índice de cobertura da população e ao diagnóstico precoce, gerando subsídios para a aplicação dos aspectos previstos no Programa de Anemia Falciforme ${ }^{(7)}$.

Com relação à prevalência de FAS, os valores se assemelharam aos encontrados em pesquisas na região Sul do Brasil, como a realizada no Rio Grande do Sul envolvendo um ano de triagem neonatal e que detectou $1,4 \%$ de alteração no padrão hemoglobínico em um total de 117.320 recém-nascidos ${ }^{(19)}$.

Na região Sudeste, outra pesquisa desenvolvida no estado de São Paulo, entre 1992 e 2000, em 78 instituições e 36 municípios, com 281.844 bebês, envolvendo a população geral encontrou prevalência de $2 \%$ de $\mathrm{HbAS}$ e $0,6 \%$ de $\mathrm{HbAC}^{(6)}$.

Uma pesquisa para detecção de hemoglobinopatia S em recém-nascidos no Instituto Materno-Infantil de referência no estado de Pernambuco identificou, em uma amostra de 1.988 sujeitos, $5,1 \%$ de prevalência do traço falciforme ${ }^{(5)}$. $O$ gene da $\mathrm{HbS}$ tem alta freqüência em toda a América, e no Brasil é mais freqüente nas regiões Sudeste e Nordeste ${ }^{(10)}$. Outro estudo, desenvolvido na cidade de Salvador, Bahia, encontrou em recém-nascidos a freqüência de $9,8 \%$ para HbAS, considerado um dos maiores valores descritos no Brasil( ${ }^{(1)}$.

A contribuição científica desse primeiro estudo de triagem realizado no estado de Mato Grosso do Sul confirma que o diagnóstico foi precoce para as alterações falciformes, como previsto no Programa de Anemia Falciforme. Nos casos de anemia falciforme, o diagnóstico precoce aponta para atendimentos ambulatoriais que favoreçam a redução da morbimortalidade em indivíduos acometidos por essas afecções.

\section{Conclusão}

A geração de informações acerca das hemoglobinopatias falciformes deste primeiro estudo realizado no Mato Grosso do Sul aponta indicadores que podem embasar ações preventivas (aconselhamento genético e estudos familiares) e assistenciais (tratamento ambulatorial contínuo), que visam à redução da morbimortalidade de indivíduos acometidos por essas afecções no estado.

\section{Agradecimentos}

Ao IPED/APAE de Campo Grande-MS agradecemos pelas informações fornecidas, que contribuíram para a realização desse estudo.

\section{Referências}

1. ADORNO, E. V. et al. Hemoglobinopathies in newborns from Salvador, Bahia, Northeast Brazil. Cad Saúde Pública, v. 21, n. 1, p. 292-8, 2005.

2. ALBERTO, F. L.; COSTA, F. F. Heterozigose para hemoglobina S. In: AGÊNCIA NACIONAL DE VIGILÂNCIA SANITÁRIA (org.). Manual de diagnóstico e tratamento das doenças falciformes. Brasília: Ministério da Saúde, 2002. p. 27-31.

3. ARAÚJO, M. C. P. E. et al. Prevalência de hemoglobinas anormais em recém-nascidos da cidade de Natal, Rio Grande do Norte, Brasil. Cad Saúde Pública, v. 20, n. 1, p. 123-8, 2004.

4. BACKES, C. E. et al. Triagem neonatal como um problema de saúde pública. Rev Bras Hematol Hemoter, v. 27, n. 1 , p. $43-7,2005$
5. BANDEIRA, F. M. G. C. et al. Características de recém-nascidos portadores de hemoglobina "S" detectados através de triagem em sangue de cordão umbilical. J Pediatr, v. 75, p. 167-71, 1999.

6. BRANDELISE, S. et al. Newborn screening for sickle cell disease in Brazil: the Campinas experience. Clin Lab Haematol, v. 26, n. 1, p.15-9, 2004.

7. BRASIL. Ministério da Saúde. Coordenação de sangue e hemoderivados. Programa de Anemia Falciforme. Portaria MS no 951, de 10/5/1996. Brasília: Ministério da Saúde, 1996. Mimeografado.

8. BRASIL. Ministério da Saúde. Programa Nacional de Triagem Neonatal. Portaria GM/MS no 822, de 7/6/2001. Brasília: Ministério da Saúde, 2001. 
9. COSTA, F. F. Anemia falciforme. In: ZAGO, M. A.; FALCÃO, R. P.; PASQUINI, R. Hematologia: fundamentos e prática. 1. ed. São Paulo: Atheneu; 2004.

10. DI NUZZO, D. V. P.; FONSECA, S. F. Anemia falciforme e infecções. J Pediatr, v. 80, n. 5, p. 347-54, 2004.

11. DOMINGOS, C. R. B. Diagnóstico laboratorial das doenças falciformes em neonatos e após o sexto mês de vida. In: Agência Nacional de Vigilância Sanitária (org.). Manual de diagnóstico e tratamento de doenças falciformes. Brasília: Ministério da Saúde, 2002. p. 19-25.

12. INSTITUTO DE PESQUISAS, ENSINO E DIAGNÓSTICOS DA ASSOCIAÇÃO DE PAIS E AMIGOS DOS EXCEPCIONAIS (IPED/APAE). Programa de proteção às gestantes. Protocolo das patologias triadas no pré-natal. (Protocolos preliminares). Campo Grande, 2003.

13. IVO, M. L.; CARVALHO, E. C. In: IVO, M. L. et al. Dimensões do processo de cuidar em enfermagem. Campo Grande: UFMS, p. 281-322, 2004.

14. KAMBLE, M.; CHATRUVEDI, P. Epidemiology of sickle cell disease in a rural hospital of central India. Indian Pediatrics, v. 37, p. 391-6, 2000.

15. NAOUM, P. C.; NAOUM, F. A. Doença das células falciformes. São Paulo: Sarvier, 2004.
16. PINHEIRO, L. S. et al. Prevalência de hemoglobina S em recém-nascidos de Fortaleza: importância da investigação neonatal. Rev Bras Ginecol Obstet, v. 28, n. 2, p. 122-5, 2006.

17. RAMALHO, A. S. et al. A Portaria MS no 822/01 e a triagem neonatal das hemoglobinopatias. Rev Bras Hematol Hemoter, v. 24, n. 4, p. 244-50, 2002.

18. SERJEANT, G. R. Sickle cell disease. Lancet, v. 350, p. 725-30, 1997.

19. SOMMER, C. K. et al. Triagem neonatal para hemoglobinopatias: experiência de um ano na rede de saúde pública do Rio Grande do Sul, Brasil. Cad Saúde Pública, Rio de Janeiro, v. 22, n. 8, p. 170914, 2006.

20. VIANA-BARACIOLI, L. M. S. et al. Prevenção de hemoglobinopatias a partir do estudo em gestantes. Rev Bras Hematol Hemoter, v. 23, n. 1, p. 31-9, 2001.

21. ZAGO, M. A. et al. Hereditary hemoglobin disorders in a brazilian population. Hum Hered, v. 33, n. 2, p.125-9, 1983.

22. ZAR, J. H. Bioestatistical analysis. 2. ed. New Jersey: Prentice Hall, 1984. 